\title{
A Decision Support Tool for Using an ICD-10 Anatomographer to Address Admission Coding Inaccuracies: A Commentary
}

\author{
Christopher M. Bell ${ }^{1,2}$, Arash Jalali ${ }^{2,3}$, Edward Mensah ${ }^{3}$
}

1 University of Illinois Hospital \& Health Sciences System, 2 University of Illinois at Chicago, Biomedical and Health Information Sciences, $\mathbf{3}$ University of Illinois at Chicago, School of Public Health

\begin{abstract}
In the chaotic environment of an emergency department trauma unit, accuracy and timeliness in decision making are required to save a patient's life. In a large urban city, where gun violence is high, emergency department physicians must have a wide array of tools in order to effectively and efficiently treat victims of gun violence and ensure that their diagnoses are properly coded. A disparity currently exists between the accuracy of ICD-9 admission coding and discharge coding with some error rates as much as seventy percent. [1,2,3,4] The elevated error rate is poised to increase even more, as the US transitions from ICD-9 to ICD-10 coding standard. The proposed decision support tool, the ICD-10 anatomographer, will have many advantages to medical professionals working in high-intensity settings. Emergency department physicians in busy trauma care units in large urban hospitals will be able to utilize this technology to find the accurate ICD-10 code in an efficient manner, thereby improving quality of care and saving lives.

Keywords: decision support, ICD-9 to ICD-10 transition, anatomography

Correspondence: chrisbel@uic.edu

Copyright @2013 the author(s)

This is an Open Access article. Authors own copyright of their articles appearing in the Online Journal of Public Health Informatics. Readers may copy articles without permission of the copyright owner(s), as long as the author and OJPHI are acknowledged in the copy and the copy is used for educational, not-for-profit purposes.
\end{abstract}

\section{The Proposed ICD-10 Anatomographer}

Much focus has been given to the pending adoption of ICD-10 coding standards in the US. The transition has been delayed for many years and many organizations are spending millions of dollars in preparing their workforces for the adoption date on October 1, 2014.[5] Coding productivity is expected to decrease ten through thirty percent for the first six months after the transition and lower levels will probably persist after that.[6] As Europe and the rest of the world await the release of ICD-11 in 2015, the US is at risk of further falling behind the international community by delaying ICD-10 adoption.[7] There is the potential for a decline in accuracy in coding when the US adopts ICD-10, but the degree to which that severity is realized will depend on the education and tools available to medical professionals. 
ICD-10 provides greater specificity and more clinical information than ICD-9, which allows for the possibility of a more accurate clinical diagnosis.[8] Physicians and coders need a tool to assist in finding the appropriate ICD-10 code in a timely manner. We propose a new visual clinical decision support tool that incorporates an anatomography software package with ICD-10 codes.

There are many anatomography software packages that render human anatomy in 3D, but currently no package incorporates ICD-10 codes into their models. Many academic institutions have been integral in developing these software packages; and as a learning tool, anatomography software packages have helped to visualize the science of anatomy.

Table 1: $\quad$ ICD-10 Anatomographer Datasets

\begin{tabular}{ll}
\hline Description & Dataset Source \\
\hline ICD-10-CM release (2013) & Centers for Medicare and Medicare Services \\
FMA Code Descriptions & University of Washington Structural Informatics Group \\
Anatomography .obj files & BodyParts3D \\
\hline
\end{tabular}

The following three datasets would be used to create the ICD-10 anatomographer: ICD-10-CM release (2013), made available by the Centers for Medicare and Medicaid Services; the FMA code descriptions, made available from the University of Washington Structural Informatics Group; and the anatomography.obj files, made available from BodyParts3D.

The use AgreementMaker, "a flexible ontology and schema matching system," [9] would allow for the ICD-10-CM dataset to be linked to the FMA code descriptions. The linked datasets would then be linked to the anatomography .obj files using ESRI ArcGIS. In order to update the existing BodyParts3D files, ESRI City Engine would be used to link the .obj files from ESRI ArcGIS. The updated anatomography .obj files would then be able to be saved to a server. 
Figure 1 ICD-10 Anatomographer Entity Relationship Diagram

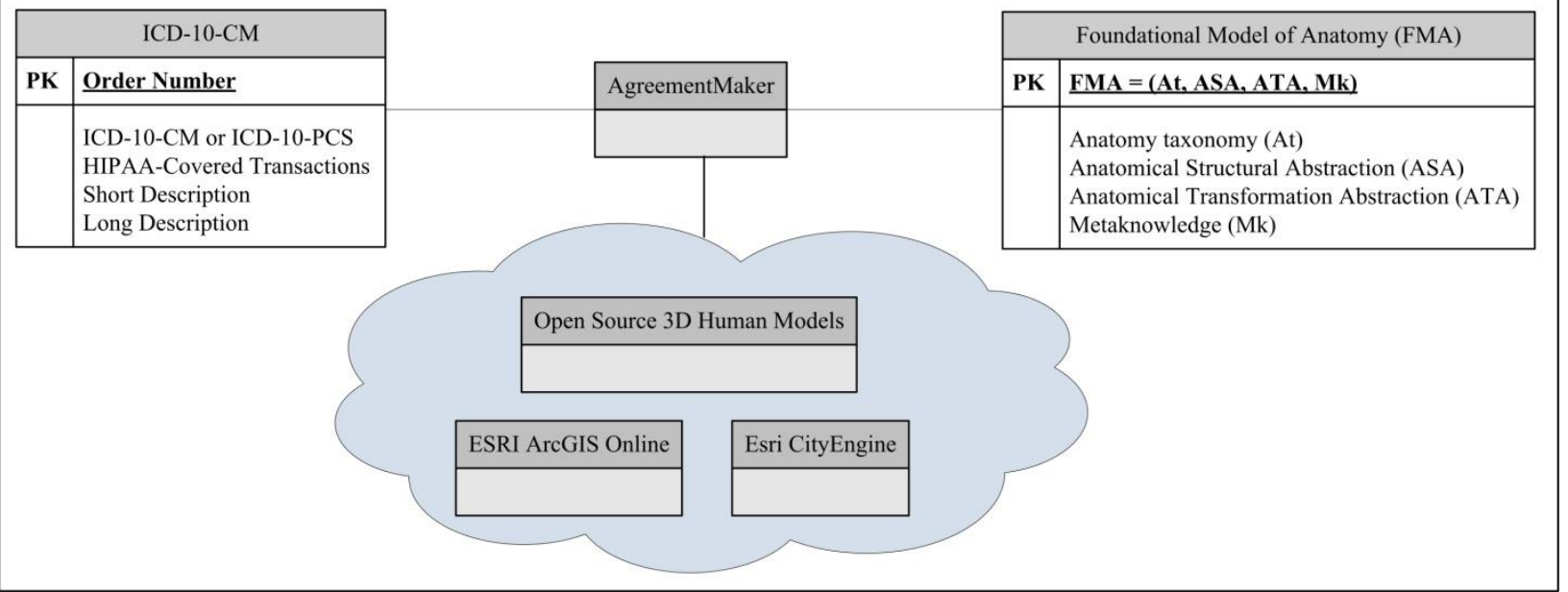

Figure 1. ICD-10 Anatomographer Entity Relationship Diagram

As a decision support tool, the proposed ICD-10 anatomographer would have many advantages to medical professionals. For emergency department physicians, the proposed ICD-10 anatomographer would help to find the accurate ICD-10 code in an efficient manner. The ICD10 anatomographer could be integrated into an existing electronic medical record as an application or as a standalone application accessible to users via the internet.

Given the current error rate for ICD-9 admission coding [1,2,3,4], the proposed ICD-10 anatomographer could be used to address many inpatient coding errors. As a visual tool the user interface of a 3D application would make finding the correct code intuitive, as codes are selected by clicking on the specific body part within the ICD-10 anatomographer application.

A preliminary proof of concept exercise performed by the authors confirms the proposed concept would be feasible. Further research and testing is needed to confirm the proposed ICD-10 anatomographer would be a viable solution to addressing the current coding inaccuracy problem.

\section{Corresponding Author}

Christopher M. Bell, CAPM

Project Coordinator

University of Illinois Hospital and Health Sciences System

Chicago, IL

Email: chrisbel@uic.edu

\section{References}

[1] O'Malley KJ, Cook KF, Price MD, Wildes KR, Hurdle JF, Ashton CM. Measuring diagnoses: ICD code accuracy. Health Services Research. 2005; 40:5p2, 1620-1639. 
[2] Benesch C, Witter DM, Wilder AL, Duncan PW, Samsa GP, Matchar DB. Inaccuracy of the International Classification of Diseases (ICD-9-CM) in identifying the diagnosis of ischemic cerebrovascular disease. Neurology. 1997; 49:3, 660-664.

[3] Faciszewski T, Broste SK, Fardon D. Quality of Data Regarding Diagnoses of Spinal Disorders in Administrative Databases. A Multicenter Study. The Journal of Bone \& Joint Surgery. 1997; 79:10, 1481-8.

[4] Goldstein LB. Accuracy of ICD-9-CM coding for the identification of patients with acute ischemic stroke effect of modifier codes. Stroke. 1998; 29:8, 1602-1604.

[5] Centers for Medicare \& Medicaid Services. ICD-10. April, 2013. Available at: http://www.cms.gov/Medicare/Coding/ICD10/index.html?redirect=/icd10. Accessed June, 2013.

[6] Carmichael A. ICD-10 and its Impact on Coder Productivity. September, 2011. Available at: http://www.icd10monitor.com/index.php?option=com_content\&id=208:icd-10-and-itsimpact-on-coder-productivity-\&Itemid=113. Accessed June, 2013.

[7] World Health Organization. The International Classification of Diseases 11th Revision is due by 2015. 2013. Available at: http://www.who.int/classifications/ icd/revision/en/. Accessed June, 2013.

[8] Hazelwood AC, Venable CA. ICD-10-CM and ICD-10-PCS Preview. AHIMA. 2004.

[9] Advances in Information System Laboratory at the University of Illinois at Chicago. AgreementMaker. May, 2012. Available at:

http://agreementmaker.org/wiki/index.php/AgreementMaker. Accessed June, 2013. 